\title{
A Biopolymer Superabsorbent Based on Alginate-Carboxymethyl Cellulose Quasi-cryogels
}

Burcu Orhan ${ }^{a, b}$, Hakan Kaygusuz ${ }^{b, c}$ *, F. Bedia Erim ${ }^{a}$

a Department of Chemistry, Faculty of Science and Letters, Istanbul Technical University, Istanbul 34469, Turkey.

${ }^{b}$ Department of Basic Sciences, Faculty of Engineering and Architecture, Altınbaş University, 34218 Mahmutbey, Istanbul, Turkey.

c Sabancı University SUNUM Nanotechnology Research Center, Istanbul 34218, Turkey.

Correspondence: hakan.kaygusuz@altinbas.edu.tr

\begin{abstract}
The research in biopolymer-based superabsorbents is gaining importance in the green chemistry field thanks to their environmentally friendly properties. In this study, a biopolymer-based superabsorbent made of calcium ion crosslinked alginate/carboxymethyl cellulose composite was prepared by a new quasi-cryogelation process, where the complete ionic gelation takes place before the freezing step, in contrast to the conventional cryogelation technique in which gelation occurs under semi-frozen conditions. Thanks to the facile quasi-cryogelation method, the morphology of the gels changed and a significant increase in water absorption properties has been achieved. The swelling properties of the material were investigated in distilled water and different physiological fluids. Results show that the proposed material shows good water absorption property where a water absorption of $2366 \%$ was reached and even after five cycles this value was $1200 \%$. As a result, this study reports a biopolymer-based sustainable superabsorbent which is appropriate for various applications such as the removal of pollutants from water or diaper production.
\end{abstract}

Keywords: alginate, carboxymethyl cellulose, superabsorbent, hydrogel, biopolymer

INTRODUCTION Biopolymers are wound dressing [7] due to their frequently used in different fields such biodegradable, biocompatible and nonas food packaging [1], removal of toxic properties compared to synthetic pollutants from water [2, 3], drug polymers. Among these, alginate (Alg) release $[4,5]$, tissue engineering [6] and is a polysaccharide obtained from the 
cell walls of brown algae and consists of covalently $(1 \rightarrow 4)$-linked blocks of $\beta$-Dmannuronate $(\mathrm{M})$ and $\alpha$-L-guluronate (G) groups. It forms a hydrogel by crosslinking in the presence of divalent cations. The solubility of alginate is dependent on the $\mathrm{pH}$ and ionic strength of the solution [8] and this property also affects the degree of swelling. Carboxymethyl cellulose (CMC), a derivative of cellulose, is another hydrophilic biopolymer. Its hydrophilicity is due to carboxymethyl moieties in its backbone is a factor for using $\mathrm{CMC}$ as an additive in superabsorbents.

Superabsorbent polymers (SAPs) can absorb water from 1 to 1000 times their mass [9]. Their main fields of use are disposable hygiene products like diapers and sanitary napkins [10, 11], self-healing concretes [12, 13], oil removal [14], agriculture [15] and dye removal [16].

Alginate/CMC is a known mixture for various applications such as drug [17] and agrochemical [18] delivery. Although there are studies on Algbased [19] and cellulose-based [20] superabsorbents, there are very few studies on Alg/CMC described as superabsorbents. Pourjavadi et al. [21] reported a superabsorbent hydrogel containing methylenebisacrylamide crosslinked Alg/CMC. In their study, the biopolymers are crosslinked using a chemical crosslinker which requires an additional synthesis step. El-Naggar [22] reported another Alg/CMC based superabsorbent. In the study, $\mathrm{N}, \mathrm{N}$ Methylene bisacrylamide (MBAM) was used as a crosslinker and $\mathrm{y}$ irradiation was applied to initiate crosslinking. In addition to chemical crosslinking by MBAM, irradiation process causes a more complicated synthesis procedure and needs extra instrument that increases the cost. Compared to these kind of covalent and ultraviolet crosslinking techniques, ionic crosslinking of alginate is more advantageous as no toxic reagents or organic solvents are used and no steps are required to remove unreacted compounds [23].

This study aimed to prove the hypothesis that it is possible to obtain an Alg/CMC superabsorbent without any covalent modification of the polymer chain with toxic chemicals. To obtain that, a green method called quasi-cryogelation was employed. Quasi-cryogelation is a complete ionic cross-linking process before freezing, mimicking the cryogelation method in which gelation occurs under semifrozen conditions. In this technique, 
gelation takes place by well-known ionic $\left(\mathrm{Ca}^{2+}\right)$ cross-linking of alginate. After the cross-linking, obtained hydrogels are frozen at $-21{ }^{\circ} \mathrm{C}$ where the retained water molecules are still inside the gel capsules. As the volume of water increases during the freezing stage, the hydrogel expands and beads with a higher surface area are obtained. The method is convenient and effective process for biopolymers, which was first applied in our previous study to enhance the dye adsorption capacity of alginate-clay composite hydrogels [24].

\section{EXPERIMENTAL}

Materials Alginic acid sodium salt (source algae (marine), $15-25 \mathrm{cP}, 1 \%$ in $\mathrm{H}_{2} \mathrm{O}$ ), carboxymethyl cellulose sodium salt (50-200 cP, $4 \%$ in $\mathrm{H}_{2} \mathrm{O}$, Degree of substitution $=0.88)$ and $\mathrm{CaCl}_{2} \cdot 2 \mathrm{H}_{2} \mathrm{O}$ were purchased from Sigma-Aldrich (St. Louis, MO). Sodium chloride and citric acid were purchased from Tekkim (Bursa, Turkey). Potassium dihydrogen phosphate was purchased from Merck (Darmstadt, Germany). pH adjustments were done by using sodium hydroxide (Sigma-Aldrich) and hydrochloric acid (Merck) solutions. All solutions were prepared with deionized water from the Rephile-Direct Pure Up system (Boston, MA).

\section{Preparation of $\mathrm{Ca}^{+2}$ crosslinked Alg/CMC beads}

According to the manufacturer's product specification sheet, the alginate used in this study has a viscosity between $15-25 \mathrm{cP}$ when it is $1 \%$ in $\mathrm{H}_{2} \mathrm{O}$. Mannuronic acid/guluronic acid ratio $(M / G)$ and molecular weight $\left(M_{w}\right)$ are not provided in the product specification sheet. However, Girón-Hernández et al. used an alginate that its viscosity ranges $15-25 \mathrm{cP}$, in $1 \% \mathrm{H}_{2} \mathrm{O}$ which is the same with the alginate used in this study [25]. The alginate with the same properties has an $M / G$ ratio of 1.56 , and the molecular weight is in the range of 120,000-190,000 g.mol-1.

A mixture was prepared by dissolving a proper amount of Alg and CMC to obtain $2 \%(\mathrm{w} / \mathrm{v})$ of both polymers at room temperature. The mixture was dropped onto a $3 \%(\mathrm{w} / \mathrm{v}) \quad \mathrm{CaCl}_{2}$ crosslinking solution using a syringe above $30 \mathrm{~cm}$. Crosslinked beads were stirred for 20 mins to obtain complete crosslinking, then filtered from the solution and washed two times with pure water to remove the excess $\mathrm{Ca}^{2+}$ ions. Beads were spread onto a Petridish and let to dry for $24 \mathrm{~h}$ at room temperature. These beads prepared with a solution containing Alg and CMC at a mass ratio of $1 / 1$ were named 
AlgCMC. The same procedure was applied with $2 \%(\mathrm{w} / \mathrm{v})$ Alg solution to prepare alginate beads as control, and these beads are named as Alg.

\section{Quasi-cryogelation process Alg/CMC} beads were prepared as described above. After the washing step, beads were put in deionized water and kept at $-21^{\circ} \mathrm{C}$ for $24 \mathrm{~h}$. Afterwards, the beads were thawed, filtered, and dried for $24 \mathrm{~h}$ at room temperature. The beads were named QuasiAlgCMC.

\section{Characterization of beads The} surface morphology of the beads was investigated using scanning electron microscopy (SEM). In the measurements, a JEOL JIB-4601 MultiBeam FIB-SEM system (Kyoto, Japan) was utilized. The Fourier transform infrared (FTIR) spectra of the biopolymers and prepared beads were obtained by a Shimadzu IRAffinity-1S (Kyoto, Japan) infrared spectrometer.

\section{Swelling experiments of the beads}

The swelling degrees of the beads were measured gravimetrically. Swelling tests were performed in deionized water, $0.9 \%$ isotonic solution, simulated intestinal fluid (SIF) at pH 6.8 containing $\mathrm{KH}_{2} \mathrm{PO}_{4}$ and $\mathrm{NaOH}$, simulated gastric fluid (SGF) at $\mathrm{pH} 1.2$ containing $\mathrm{NaCl}$ and $\mathrm{HCl}$, and citrate-phosphate buffer saline (Citrate-PBS) at $\mathrm{pH} 5.5$ solutions containing citric acid. SIF, SGF and citrate-PBS solutions were without enzymes. Alg, AlgCMC and QuasiAlgCMC beads were separately taken $0.1 \mathrm{~g}$ and submerged into swelling solutions. Incubated beads were swollen in a water-bath of Nüve ST-30 (Ankara, Turkey) at $37^{\circ} \mathrm{C}$ and 90 rpm shaking rate. Beads were filtered from their solution at specific time intervals, and the excess solution was gently removed using filter paper. Then, beads were weighed and submerged back. The experiment was replicated three times for each type of bead. Percentage swelling is calculated according to the following equation:

Swelling $(\%)=\frac{m_{f}-m_{i}}{m_{i}} \times 100$

Where $m_{f}$ and $m_{i}$ define the mass of swollen bead and the mass of dried bead, respectively.

\section{Deswelling and reusability tests of} the beads Pre-weighted $0.016 \mathrm{~g}$ of QuasiAlgCMC beads were swollen in deionized water and isotonic solution for $4 \mathrm{~h}$ to achieve their maximum swelling degree in shaking water bath at $37^{\circ} \mathrm{C}$. Swollen beads were extracted from the solution and dried at $120{ }^{\circ} \mathrm{C}$ with a moister analyzer of Shimadzu MOC63u (Kyoto, Japan). Dried mass 
was read from the instrument and recorded. The same beads were treated with the same procedure to obtain five cycles. Swelling capacity is calculated according to the following equation:

Swelling capacity $=\frac{m_{f}-m_{i}}{m_{i}}$

Swelling kinetic study Kinetic behavior of the QuasiAlgCMC in different solutions were modelled pseudo-first order and pseudo-secondorder kinetic models. Pseudo-second order kinetic model was found to be suitable for the data $\left(R^{2}>0.99\right)$, where the $R^{2}$ value for pseudo-first order was below 0.90 . Therefore, the pseudokinetic model, which represented in the following equation was used in the kinetic modeling:

$\frac{t}{Q_{t}}=\frac{1}{k_{2} Q_{e}^{2}}+\frac{t}{Q t}$

Here $Q_{e}(\mathrm{~g} / \mathrm{g})$ and $Q_{t}(\mathrm{~g} / \mathrm{g})$ are the swelling values (absorbed amount of solution) in equilibrium and time $t$, respectively. $K_{2}$ is the rate constant for the pseudo-second-order kinetic model [26].

\section{RESULTS AND DISCUSSION}

\section{Swelling ratio of beads}

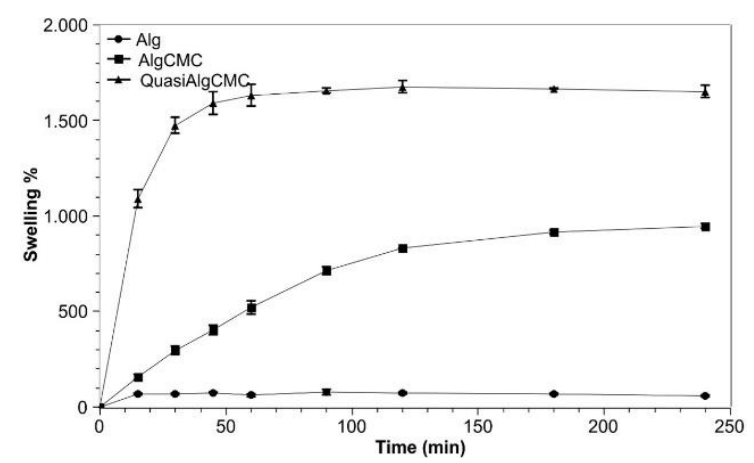

Fig. 1 Swelling ratios of Alg, AlgCMC and QuasiAlgCMC beads tested in pure water

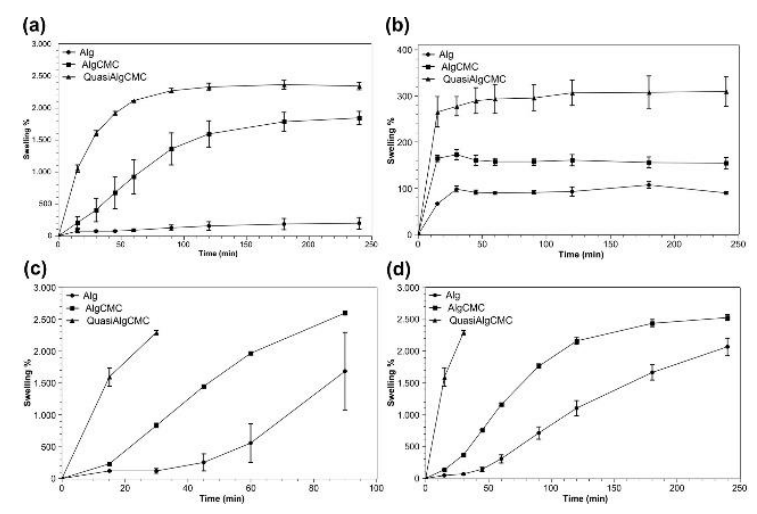

Fig. 2 Swelling ratios of Alg, AlgCMC and QuasiAlgCMC beads in different swelling solutions: $0,9 \%(\mathrm{w} / \mathrm{v})$ isotonic solution (a), SGF (b), SIF (c) and Citrate-PBS (d), respectively

Alginate hydrogels have wide applications in the drug release systems. Drug release occurs as hydrogel swells. Drug release system may be directed to any part of the body including the stomach, gut, and skin. Therefore, in addition to pure water, four different solutions such as isotonic, SGF, SIF and citrate-PBS were used as 
swelling solutions to provide a basis for drug release applications. Those solutions are supposed to simulate cell osmolarity, stomach medium, gut medium, and skin medium, respectively.

Fig. 1 and Fig. 2 show the graphs comparing the swelling degrees of Alg, AlgCMC and QuasiAlgCMC beads in pure water (Fig. 1) and in isotonic, SGF, SIF and citrate-PBS solutions (Fig. 2).

As seen in Fig. 1 and Fig. 2, the swelling degree was the lowest for Alg, followed by AlgCMC and the highest for QuasiAlgCMC beads in each solution. Alg beads swelled $56 \%$ in pure water, $197 \%$ in isotonic solution, $90 \%$ in SGF, $1683 \%$ in SIF and $2066 \%$ in citratePBS. The addition of CMC resulted in higher swelling degrees that their values are mentioned further on.

Alginate and $\mathrm{CMC}$ are $\mathrm{pH}$-sensitive polyanionic polymers. Under acidic conditions where $\mathrm{pH}$ is below $\mathrm{pKa}$ of alginate (3.5) and CMC (3-4), protonation of - $\mathrm{COO}^{-}$groups occurs, and hydrophilic character decreases due to the decreased number of negative charges on the polymer chain, consequently swelling decreases. In the exact opposite conditions, dissociation of $-\mathrm{COOH}$ groups occurs and swelling increases due to repulsion of negative charges and expansion of the hydrogel
[27]. Based on this, as seen in Fig. 2, AlgCMC beads swelled least in SGF $(\mathrm{pH} 1.2)$ solution with a swelling degree of $160 \%$ and most in SIF and citratePBS with swelling degrees of $2526 \%$ and $2598 \%$. However, beads degraded after 90 mins in SIF and 240 mins in citrate-PBS. It is thought to be caused by the affinity of phosphate ions to $\mathrm{Ca}^{2+}$ ions. With their interaction, phosphate withdraws $\mathrm{Ca}^{2+}$ toward itself and causes breakage of crosslinks. AlgCMC beads swelled $959 \%$ in pure water and $1847 \%$ in isotonic solution because of neutral $\mathrm{pH}$ above their $\mathrm{pKa}$ values. Ionic strength is the reason for more swelling in isotonic solution even if $\mathrm{pH}$ values are the same. The other reason also can be explained by the salting-in phenomenon [19]. The phenomenon states that the charge of polymer increases in the existence of salt ions and promotes enhancing absorbency. Studies where alginate has a higher swelling ratio in saline solutions compared to pure water have been reported. Mallepaly et al. reported an alginate aerogel that swells $120 \mathrm{~g} / \mathrm{g}$ in saline solution and $20 \mathrm{~g} / \mathrm{g}$ in deionized water [19]. Moreover, Mikula et al. reported that calcium crosslinked alginate hydrogel changed its weight $10 \%$ in pure water and $90 \%$ in isotonic solution [28]. In addition to this, in the presence of sodium ions cation 
crosslinked alginate chains tend to convert into sodium alginate by ionexchange mechanism, and sodium alginate tends to absorb high quantity of water [29]. However, AlgCMC beads did not degrade in the isotonic solution, unlike Mikula et al. [28] reported.

QuasiAlgCMC beads substantially enhanced the swelling of AlgCMC beads in each solution as shown in Fig. 1 and Fig. 2. In pure water, isotonic and SGF solutions, swelling enhanced from $959 \%$ to $1677 \%$, from $1847 \%$ to $2366 \%$ and from $160 \%$ to $309 \%$, respectively by quasi-cryogelation. In SIF and citrate-PBS solutions, QuasiAlgCMC beads degraded in 30 mins due to the phosphate effect occurring quickly because of rapid swelling. Fig. 3 shows the images of dried and swollen QuasiAlgCMC beads in pure water. As can be seen in the figure, unlike the ordinary alginate beads, quasi-cryogels have a snowflake-like appearance in their dried form. However, in the swollen form, they regain the spherical shape.

Since SIF solution ( $\mathrm{pH} \mathrm{6.8)}$ is more basic than citrate-PBS solution $(\mathrm{pH}$ 5.5), AlgCMC and Alg beads degraded earlier in SIF (90 mins) compared to citrate-PBS (240 min). Under basic conditions where $\mathrm{pH}$ is above $\mathrm{pKa}$ of alginate (3.5) and CMC (3-4), dissociation of $-\mathrm{COOH}$ groups occurs, and swelling increases due to repulsion of negative charges and expansion of the hydrogel. So, the early degradation of AlgCMC and Alg beads in SIF is thought to be caused by both the basicity and phosphate effect. (a)

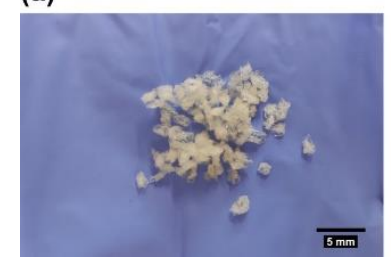

(b)

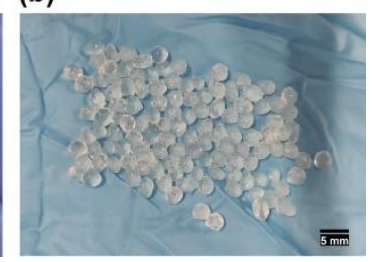

Fig. 3 The photos of QuasiAlgCMC beads before swelling test treatment (a) and after being swollen (b) in pure water

\section{Deswelling and reusability of beads}

An important property of adsorbents is reusability due to environmental and economic concerns. They are reusable if the adsorbents exhibit similar performance when repeatedly swelled and dried.

Fig. 4 shows the graph swelling capacity against the number of uses in pure water. QuasiAlgCMC beads maintain their swelling capacity with a minimal decrease until the third cycle but later begin to decrease in pure water. Starting from $\sim 22 \mathrm{~g} / \mathrm{g}(\sim \% 2200)$, the capacity was $12 \mathrm{~g} / \mathrm{g}(\sim \% 1200)$ even at the end of the fifth cycle, and no structural alteration was detected on the beads. The test was performed also in 
the isotonic solution. The swelling capacity in isotonic solution was $\sim 29 \mathrm{~g} / \mathrm{g}$ ( \%2900). Although the decrease in capacity between the first and second cycles was from $29.75 \mathrm{~g} / \mathrm{g}$ to $29.42 \mathrm{~g} / \mathrm{g}$, the beads degraded at the 3rd cycle and became unusable.

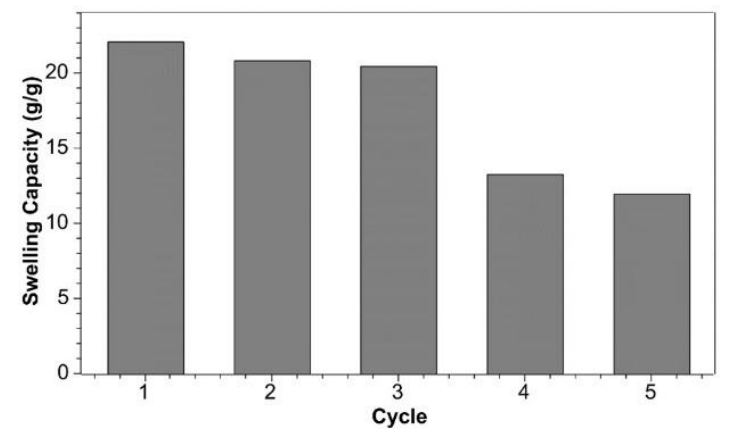

Fig. 4 Changing in swelling capacity against the number of uses in pure water related to deswelling \& reusability test

\section{Characterization of beads}

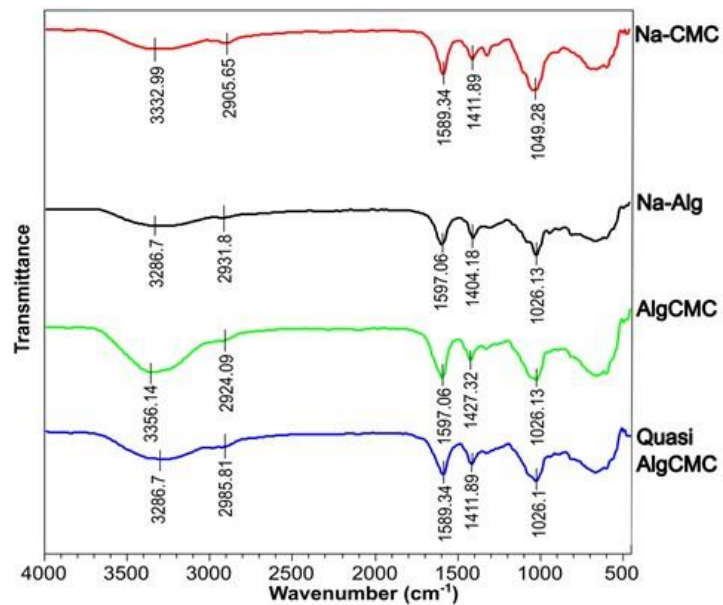

Fig. 5 FT-IR spectra of alginic acid sodium salt (Na-Alg), carboxymethyl cellulose sodium salt (Na-CMC), AlgCMC and QuasiAlgCMC beads

Fig. 5 shows the FT-IR spectrums of four compounds: sodium alginate powder, sodium CMC powder,

AlgCMC, and QuasiAlgCMC beads. Characteristic peaks of alginate have been seen as $\mathrm{O}-\mathrm{H}$ stretch at $3286 \mathrm{~cm}^{-1}$, $\mathrm{C}-\mathrm{H}$ stretch at $2931 \mathrm{~cm}^{-1}$, antisymmetric $\mathrm{CO}_{2}^{-}$stretch at $1597 \mathrm{~cm}^{-1}$, symmetric $\mathrm{CO}_{2}^{-}$stretch at $1404 \mathrm{~cm}^{-1}$ and antisymmetric C-O-C stretch at 1026 $\mathrm{cm}^{-1}$ [30]. Besides small shifts, these characteristic peaks are also present for $\mathrm{CMC}$ as it has $\mathrm{O}-\mathrm{H},-\mathrm{CO}_{2}^{-}, \mathrm{C}-\mathrm{O}-\mathrm{C}$, and $\mathrm{C}-\mathrm{H}$ bonds either. The stretching vibration peaks of $\mathrm{O}-\mathrm{H}$ of AlgCMC and QuasiAlgCMC beads were more intense than the pure powder form of biopolymers which demonstrates that there may be hydrogen bonds between alginate and CMC [31]. It is approved that alginate and CMC are not covalently bonded due to the lack of new peaks except broadening or shifting in the AlgCMC sample. No significant difference has been observed between AlgCMC and QuasiAlgCMC spectra since quasicryogelation is a physical process instead of a chemical modification.

(a)

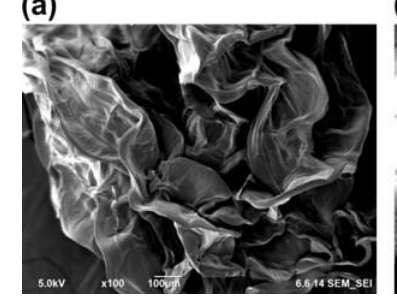

Fig. 6 SEM images of QuasiAlgCMC (a) and AlgCMC (b) beads (b)

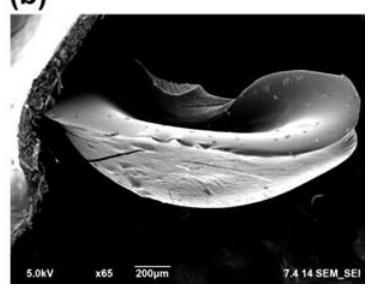
C

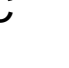


Fig. 6 shows SEM images of AlgCMC and QuasiAlgCMC beads. When compared to AlgCMC, the QuasiAlgCMC bead was much more complex and sponge-like. The increase in the volume of beads due to the freezing of entrapped hydrogel moisture resulted in cracking and a higher surface area of the beads, where a similar result was also observed previously for alginate-clay and alginate-graphene oxide gels obtained by quasi-cryogelation [24, 32]. (a)

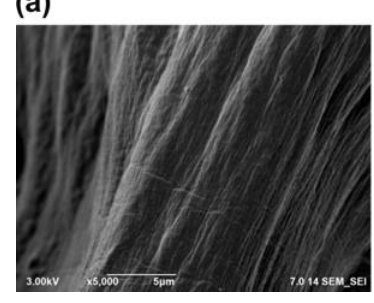

(b)

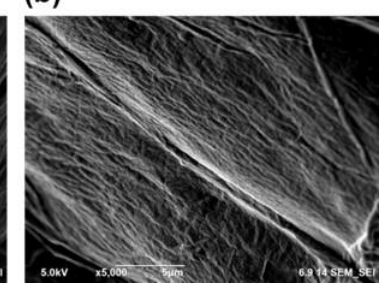

Fig. 7 SEM images of QuasiAlgCMC beads before (a) and after (b) deswelling \& reusability test

Fig. 7 shows the morphology of the same QuasiAlgCMC bead before and after the 5th cycle. Besides the additional fractures, the surface kept its integrity at the end of the fifth cycle, which explains the adsorption capacity is still above $10 \mathrm{~g} / \mathrm{g}$ at the end of the fifth cycle.

As for the superabsorbent characterization, a hydrogel that can absorb deionized water at a range of
$10-1000 \mathrm{~g} / \mathrm{g}$ is defined as a superabsorbent [33]. Since the QuasiAlgCMC beads have $\sim 22 \mathrm{~g} / \mathrm{g}$ in deionized water (and $12 \mathrm{~g} / \mathrm{g}$ after five cycles) and $\sim 29 \mathrm{~g} / \mathrm{g}$ in sodium chloride solution, the QuasiAlgCMC beads can be classified as a superabsorbent. Therefore, it is proven that it is possible to obtain Alg/CMC superabsorbents without any chemical crosslinking. The results of the pseudo-second order model is shown in Figure 8 and the parameters are summarized Table 1. $R^{2}$ value of the model is very high for all of the solutions listed in Table 1, therefore pseudo-second order model can be used for representing the swelling kinetics of the QuasiAlgCMC. Additionally, the calculated $Q_{e}$ values are very close to the measured $Q_{e}$ values. The data in SIF and PBS are not included in results, since the swelling is very fast in these two medium and early degradations were observed.

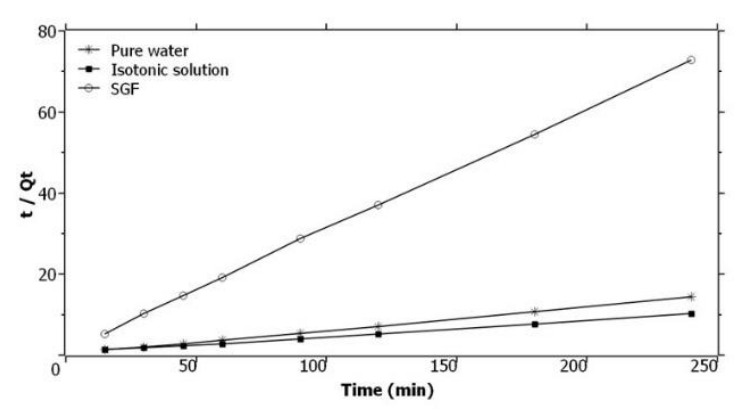

Fig. 8 Pseudo-second-order kinetic plot for the swelling of QuasiAlgCMC 
beads in pure water, isotonic $\mathrm{NaCl}$ solution and SGF

Table 1 Results of the pseudo-secondorder kinetic model for QuasiAlgCMC beads in pure water, isotonic $\mathrm{NaCl}$ solution and SGF

\begin{tabular}{c|c|c|c|c} 
& \multirow{2}{*}{$\begin{array}{c}\text { Experimental } Q_{\mathrm{e}}(\mathrm{g} / \mathrm{g}) \\
\text { (Swelling capacity) }\end{array}$} & \multicolumn{3}{|c}{ Pseudo second order model } \\
\cline { 3 - 5 } & & $K_{2}$ & $Q_{\mathrm{g}}(\mathrm{g} / \mathrm{g})$ & $R^{2}$ \\
\hline Pure water & 16.77 & 0.014 & 17.14 & 0.999 \\
\hline Isotonic & 23.66 & 0.003 & 25.50 & 0.997 \\
solution & & & & \\
\hline SGF & 3.09 & 0.07 & 3.36 & 0.999
\end{tabular}

\section{CONCLUSION}

A polymer solution containing $2 \%(\mathrm{w} / \mathrm{v})$ Alg and 2\% (w/v) CMC (AlgCMC) was crosslinked with calcium to prepare hydrogel beads. These beads were later modified to QuasiAlgCMC beads by deep-freezing at $-21{ }^{\circ} \mathrm{C}$ called the quasi-cryogelation method. The swelling degree of the beads was tested in distilled water and different physiological fluids. In each swelling solution, CMC addition to Alg rose the swelling degree. The swelling percentage of ionically cross-linked beads dramatically increased after the freezing process due to a significant change of the morphologies of the beads. QuasiAlgCMC beads swelled $1677 \%$, 2366\%, 309\% in pure water, isotonic solution and SGF, respectively. The behaviour in pure water, isotonic solution and SGF provides a potential for drug delivery in osmotic and stomach medium. Additionally, the beads swelled $2318 \%$ in citrate-PBS and $2294 \%$ in SIF however they degraded in 30 mins. A good reusability of the beads was obtained with almost constant swelling capacity around 22 $\mathrm{g} / \mathrm{g}$ after three cycles. Based on these results, it is proven that QuasiAlgCMC beads were successfully prepared and have superabsorbent properties.

\section{Acknowledgements}

The results of this study are derived from the M.Sc thesis of Burcu Orhan. The authors would like to thank Istanbul Technical University Capillary Electrophoresis and Biopolymer Applications Research Laboratory and Sabancı University SUNUM for the supply of materials and providing the opportunities for testing.

\section{REFERENCES}

1. Capello C, Leandro GC, Gagliardi TR, Valencia GA (2021) Intelligent Films from Chitosan and Biohybrids Based on Anthocyanins and Laponite $®$ : Physicochemical Properties and Food Packaging Applications. J Polym Environ 29:3988-3999. https://doi.org/10.1007/s10924021-02168-5

2. Kaygusuz $H$, Uzaşçı $S$, Erim FB (2015) Removal of Fluoride from Aqueous Solution Using Aluminum Alginate Beads. CLEAN - Soil, Air, Water 43:724- 
730.

https://doi.org/10.1002/clen.2013 00632

3. Atangana E, Oberholster PJ (2020) Modified Biopolymer (Chitin-Chitosan Derivatives) for the Removal of Heavy Metals in Poultry Wastewater. J Polym Environ 28:388-398. https://doi.org/10.1007/s10924019-01616-7

4. Kaygusuz $\mathrm{H}$, Uysal $\mathrm{M}$, Adımcılar V, Erim FB (2015) Natural alginate biopolymer montmorillonite clay composites for vitamin B2 delivery. J Bioact Compat Polym 30:48-56. https://doi.org/10.1177/08839115 14557014

5. Kaygusuz H, Erim FB (2013) Alginate/BSA/montmorillonite composites with enhanced protein entrapment and controlled release efficiency. React Funct Polym 73:1420-1425. https://doi.org/10.1016/j.reactfun ctpolym.2013.07.014

6. Lin CC, Chiu JY (2021) Glycerolmodified $Y$-PGA and gellan composite hydrogel materials with tunable physicochemical and thermal properties for soft tissue engineering application. Polymer 230:124049.

https://doi.org/10.1016/J.POLYM ER.2021.124049

7. Kalaycıoğlu Z, Kahya N, Adımcılar V, et al (2020) Antibacterial nano cerium oxide/chitosan/cellulose acetate composite films as potential wound dressing. Eur Polym J 133:109777.

https://doi.org/10.1016/j.eurpoly mj.2020.109777

8. Donati I, Paoletti S (2009) Material Properties of Alginates. Springer, Berlin, Heidelberg, pp
$1-53$

9. Horie K, Barón M, Fox RB, et al (2004) Definitions of terms relating to reactions of polymers and to functional polymeric materials:

(IUPAC Recommendations 2003). Pure Appl. Chem. 76:889-906

10. G R, C.R R, Nair S V., Menon D (2020) Superabsorbent sodium carboxymethyl cellulose membranes based on a new cross-linker combination for female sanitary napkin applications. Carbohydr Polym 248:116763.

https://doi.org/10.1016/J.CARBP OL.2020.116763

11. Shahi S, Motasadizadeh HR, Zohuriaan-Mehr MJ (2017) Surface modification of superabsorbing hydrogels through a feasible esterification reaction: Toward tunable superabsorbent for hygienic applications. Int J Polym Mater Polym Biomater 66:544-557. https://doi.org/10.1080/00914037 .2016 .1252348

12. Mignon A, Graulus GJ, Snoeck D, et al (2014) pH-sensitive superabsorbent polymers: a potential candidate material for self-healing concrete. J Mater Sci 50:970-979. https://doi.org/10.1007/s10853014-8657-6

13. Snoeck D, Van Tittelboom K, Steuperaert S, et al (2014) Selfhealing cementitious materials by the combination of microfibres and superabsorbent polymers. J Intell Mater Syst Struct 25:13-24. https://doi.org/10.1177/1045389 X12438623

14. Loh JW, Goh XY, Nguyen PTT, et al (2022) Advanced Aerogels from Wool Waste Fibers for Oil 
Spill Cleaning Applications. J Polym Environ 30:681-694. https://doi.org/10.1007/s10924021-02234-y

15. Sharma J, Sukriti, Kaith BS, Bhatti MS (2018) Fabrication of Biodegradable Superabsorbent Using RSM Design for Controlled Release of KNO3. J Polym Environ 26:518-531. https://doi.org/10.1007/s10924017-0959-8

16. Feng $D$, Bai $B$, Wang $H$, Suo $Y$ (2018) Novel Fabrication of PAA/PVA/Yeast Superabsorbent with Interpenetrating Polymer Network for pH-Dependent Selective Adsorption of Dyes. J Polym Environ 26:567-588. https://doi.org/10.1007/s10924017-0972-y

17. Bulut E, Şanli O (2014) Novel ionically crosslinked acrylamidegrafted poly(vinyl alcohol)/sodium alginate/sodium carboxymethyl cellulose $\quad \mathrm{pH}$-sensitive microspheres for delivery of Alzheimer's drug donepezil hydrochloride: Preparation and optimization of release conditions. Artif Cells Nanomed Biotechnol 44:431-442. https://doi.org/10.3109/21691401 .2014 .962741

18. Bajpai J, Mishra S, Bajpai AK (2007) Dynamics of controlled release of potassium nitrate from a highly swelling binary polymeric blend of alginate and carboxymethyl cellulose. J Appl Polym Sci 106:961-972. https://doi.org/10.1002/APP.2670 3

19. Mallepally RR, Bernard I, Marin MA, et al (2013) Superabsorbent alginate aerogels. J Supercrit Fluids 79:202-208. https://doi.org/10.1016/j.supflu.2
012.11 .024

20. Demitri C, Del Sole R, Scalera F, et al (2008) Novel superabsorbent cellulose-based hydrogels crosslinked with citric acid. J Appl Polym Sci 110:24532460.

https://doi.org/10.1002/app.2866 0

21. Pourjavadi A, Barzegar $S$, Mahdavinia GR (2006) MBAcrosslinked $\mathrm{Na}-\mathrm{Alg} / \mathrm{CMC}$ as a smart full-polysaccharide superabsorbent hydrogels. Carbohydr Polym 66:386-395. https://doi.org/10.1016/j.carbpol. 2006.03.013

22. El-Naggar AA (2016) Radiation synthesis of superabsorbent hydrogels based on carboxymethyl cellulose/sodium alginate for absorbent of heavy metal ions from waste water. $J$ Thermoplast Compos Mater 29:16-27.

https://doi.org/10.1177/08927057 13518786

23. Nataraj D, Reddy N (2020) Chemical modifications of alginate and its derivatives. Int $\mathrm{J}$ Chem Res 1-17. https://doi.org/10.22159/IJCR.20 20V4I1.98

24. Uyar G, Kaygusuz H, Erim FB (2016) Methylene blue removal by alginate-clay quasi-cryogel beads. React Funct Polym 106:1-7.

https://doi.org/10.1016/j.reactfun ctpolym.2016.07.001

25. Girón-Hernández J, Gentile $P$, Benlloch-Tinoco M (2021) Impact of heterogeneously crosslinked calcium alginate networks on the encapsulation of $\beta$-caroteneloaded beads. Carbohydr Polym 271:118429. https://doi.org/10.1016/J.CARBP 
OL.2021.118429

26. Ho YS, McKay G (1999) Pseudosecond order model for sorption processes. Process Biochem 34:451-465.

https://doi.org/10.1016/S00329592(98)00112-5

27. Kahya N, Erim FB (2019) Surfactant modified alginate composite gels for controlled release of protein drug. Carbohydr Polym 224:115165. https://doi.org/10.1016/j.carbpol. 2019.115165

28. Mikula K, Skrzypczak D, Ligas B, Witek-Krowiak A (2019) Preparation of hydrogel composites using $\mathrm{Ca} 2+$ and $\mathrm{Cu} 2+$ ions as crosslinking agents. SN Appl Sci 1:1-15. https://doi.org/10.1007/s42452019-0657-3

29. Riaz S, Malik S, Hussain T, et al (2018) Development of antibacterial fibers and study on effect of guar-gum addition on properties of carboxymethylcellulose (CMC)/alginate fibers. IOP Conf Ser Mater Sci Eng 414:012020. https://doi.org/10.1088/1757899X/414/1/012020

30. Gwen Lawrie, Imelda Keen, Barry Drew, et al (2007) Interactions between Alginate and Chitosan Biopolymers Characterized Using FTIR and XPS. Biomacromolecules 8:2533-2541.

https://doi.org/10.1021/BM07001 4Y

31. Ren H, Gao Z, Wu D, et al (2016) Efficient $\mathrm{Pb}(\mathrm{II})$ removal using sodium alginate-carboxymethyl cellulose gel beads: Preparation, characterization, and adsorption mechanism. Carbohydr Polym 137:402-409.
https://doi.org/10.1016/J.CARBP OL.2015.11.002

32. Balkız G, Pingo E, Kahya N, et al (2018) Graphene Oxide/Alginate Quasi-Cryogels for Removal of Methylene Blue. Water Air Soil Pollut 229:1-9. https://doi.org/10.1007/s11270018-3790-5

33. M.J.A.D. ZM, K. K (2008) Superabsorbent polymer materials: a review. Iran. Polym. J. 17:451-447 